\title{
Australia-Modified Karnofsky Performance Status 40
}

National Cancer Institute

\section{Source}

National Cancer Institute. Australia-Modified Karnofsky Performance Status 40. NCI

Thesaurus. Code C107490.

In bed more than $50 \%$ of the time. 